\title{
Wound Protectors in Reducing Surgical Site Infections in Lower Gastrointestinal Surgery: An Updated Meta-Analysis
}

\section{Lisa Zhang, Basheer Elsolh, Sunil V. Patel}

Background: Surgical site infection (SSI) is a common

complication in gastrointestinal surgery

Objective: To determine if wound protectors reduce the incidence of SSIs in lower gastrointestinal surgery

Study Design: Meta-analysis

Study Selection: RCTs comparing wound protector to no wound protector

Primary Outcome: SSI

\begin{tabular}{|c|c|c|c|c|}
\hline Study & $\mathbf{N}$ & $\begin{array}{l}\text { Type of } \\
\text { Surgery }\end{array}$ & $\begin{array}{l}\text { Wound } \\
\text { Protector }\end{array}$ & Follow Up \\
\hline Baier 2012 & 199 & Colorectal & Single Ring & 30 days \\
\hline Cheng 2012 & 64 & Colorectal & Dual Ring & 30 days \\
\hline Horiuchi 2007 & 221 & $\begin{array}{l}\text { Colorectal, } \\
\text { HPB, Gastric }\end{array}$ & Dual Ring & 30 days \\
\hline Lauscher 2012 & 93 & Colorectal & Single Ring & 6 months \\
\hline Lee 2009 & 109 & Appendix & Dual Ring & 21 days \\
\hline Mihaljevic 2014 & 594 & $\begin{array}{l}\text { Colorectal, } \\
\text { SB \& HPB, } \\
\text { Gastric }\end{array}$ & Single Ring & $30-45$ days \\
\hline Nystrom 1984 & 140 & Colorectal & Single Ring & 30 days \\
\hline Pinkney 2013 & 749 & $\begin{array}{l}\text { Colorectal, } \\
\text { SB \& HPB, } \\
\text { Gastric }\end{array}$ & Single Ring & 30-33 days \\
\hline Psaila 1977 & 144 & Colorectal & Single Ring & >3 days \\
\hline Reid 2010 & 130 & Colorectal & Dual Ring & 30 days \\
\hline Silva 2008 & 433 & Appendix & Dual Ring & 30 days \\
\hline Williams 1972 & 167 & $\begin{array}{l}\text { Colorectal, } \\
\text { SB \& HPB, } \\
\text { Gastric }\end{array}$ & Single Ring & 7-10 days \\
\hline
\end{tabular}

\section{Risk of Bias}

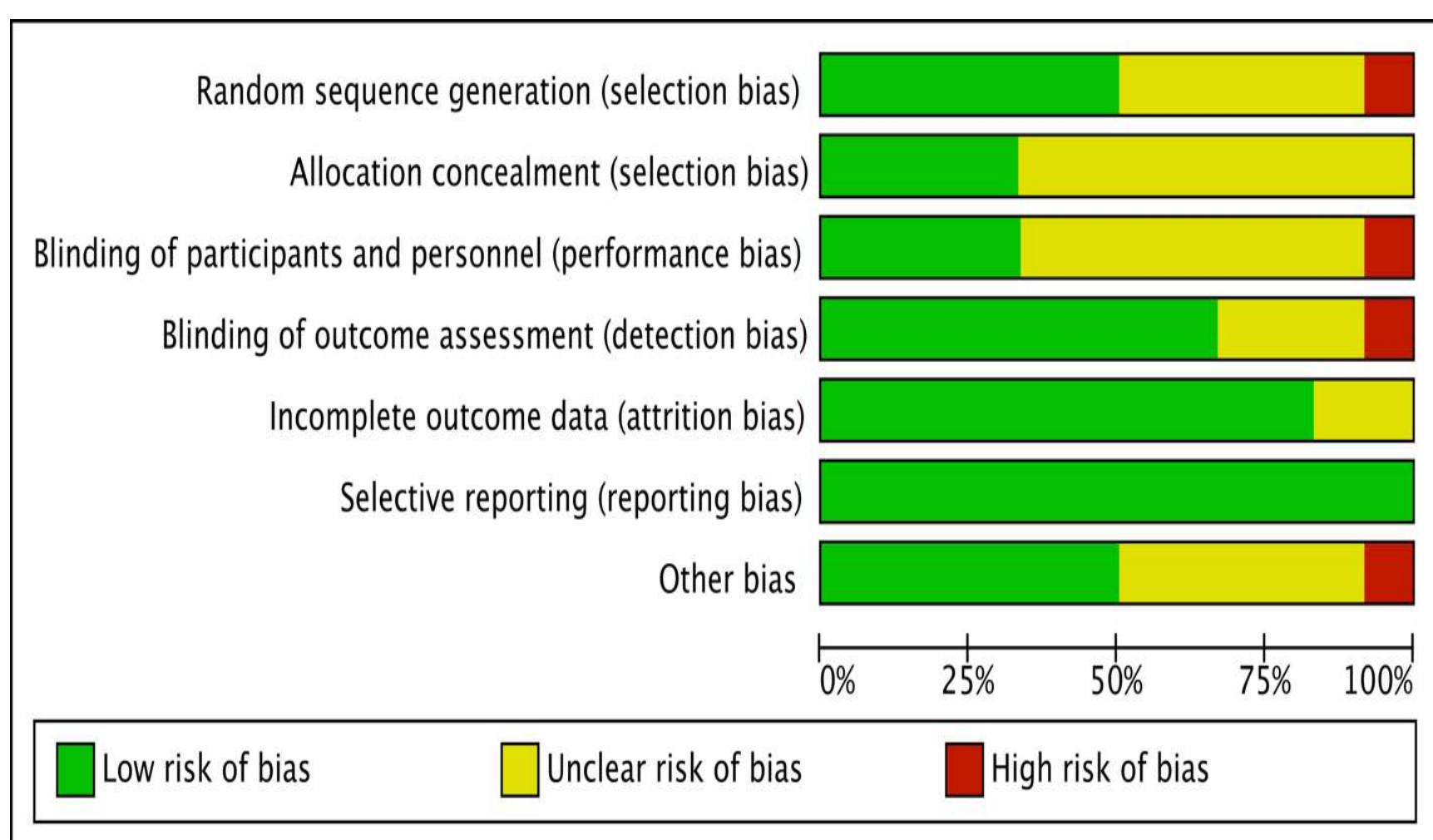

\section{Wound Protector vs. No Wound Protector}

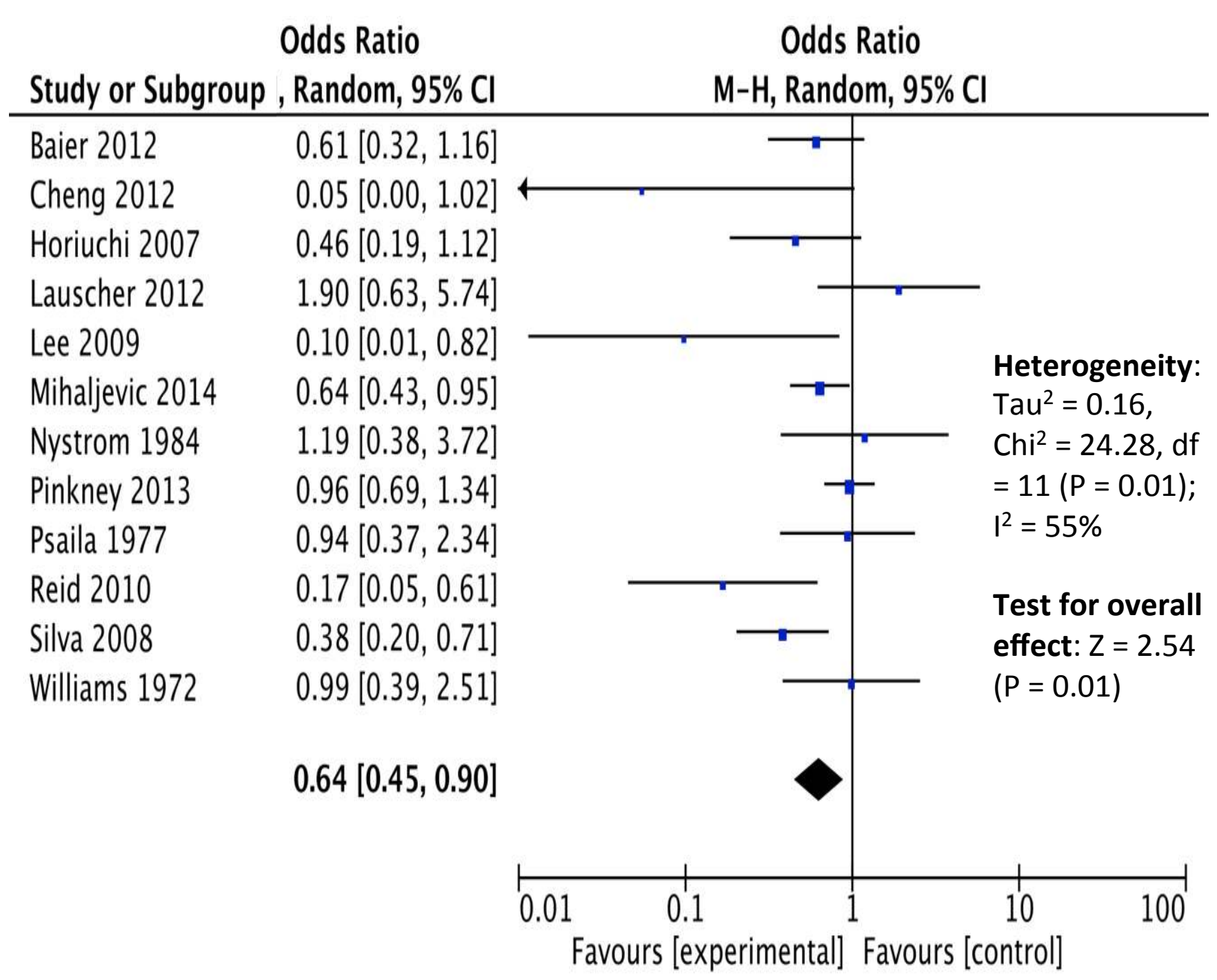

\begin{tabular}{|c|c|c|}
\hline Subgroup Analysis & $\begin{array}{l}\text { Odds Ratio } \\
\text { (95\% CI) }\end{array}$ & $\begin{array}{c}\text { Test of } \\
\text { Subgroup } \\
\text { Difference }\end{array}$ \\
\hline \multicolumn{3}{|l|}{ Wound Protector } \\
\hline $\begin{array}{l}\text { Single Ring ( } 7 \text { Studies, N = } \\
\text { 2072) } \\
\text { Dual Ring ( } 5 \text { Studies, N = } \\
\text { 957) }\end{array}$ & $0.84(0.67,2.04)$ & $P<0.0001$ \\
\hline \multicolumn{3}{|l|}{ Type of Surgery } \\
\hline $\begin{array}{l}\text { Colorectal ( } 8 \text { Studies, } N= \\
1352 \text { ) }\end{array}$ & $0.56(0.33,0.96)$ & \multirow{3}{*}{$P=0.12$} \\
\hline $\begin{array}{l}\text { Appendix (2 Studies, } N= \\
542 \text { ) }\end{array}$ & $0.29(0.10,0.86)$ & \\
\hline Other (4 Studies, $N=828$ ) & $0.84(0.59,1.18)$ & \\
\hline
\end{tabular}

Conclusions: Wound protector use is associated with decreased odds of developing SSI in patients undergoing lower gastrointestinal surgery. There was a subgroup effect when comparing dual ring to single ring devices.

This study is limited by the high risk of bias in 3 of the included RCTs.

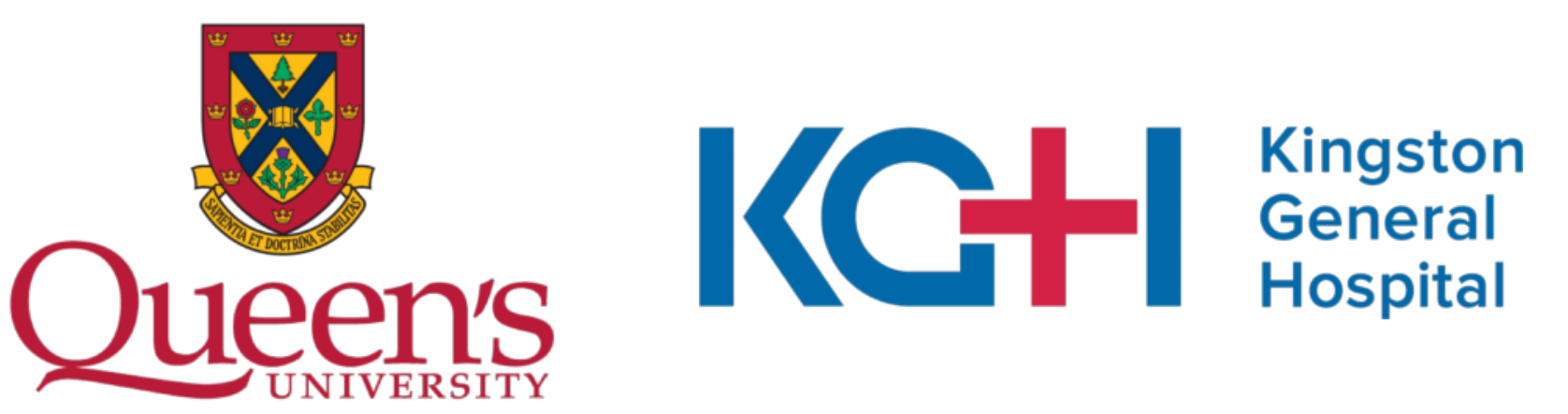

